\title{
Association between internal carotid artery dissection and arterial tortuosity
}

\author{
Luca Saba • Giovanni Maria Argiolas • Suna Sumer • \\ Paolo Siotto • Eytan Raz • Roberto Sanfilippo • \\ Roberto Montisci • Mario Piga • Max Wintermark
}

Received: 4 June 2014 / Accepted: 18 September 2014

(C) Springer-Verlag Berlin Heidelberg 2014

\begin{abstract}
Introduction Carotid artery dissection is an important cause of ischemic stroke in all age groups, particularly in young patients. The purpose of this work was to assess whether there is an association between the presence of an internal carotid artery dissection (ICAD) and the arterial tortuosity.

Methods This study considered 124 patients ( 72 males and 52 females; median age 57 years) with CT/MR diagnosis of ICAD of the internal carotid artery were considered in this multi-centric retrospective study. The arterial tortuosity was evaluated and, when present, was categorized as elongation, kinking, or coiling. For each patient, both the right and left sides were considered for a total number of 248 arteries in order to have the same number of cases and controls. Fisher's exact test was applied to test the association between
\end{abstract}

\section{Saba $(\square) \cdot$ M. Piga}

Department of Radiology, Azienda Ospedaliero Universitaria

(A.O.U.), di Cagliari - Polo di Monserrato s.s. 554,

09045 Monserrato, Cagliari, Italy

e-mail: lucasaba@tiscali.it

G. M. Argiolas $\cdot$ P. Siotto

Department of Radiology, Azienda Ospedaliero Brotzu (A.O.B.), di Cagliari, Italy

S. Sumer $\cdot$ M. Wintermark

Neuroradiology, UVA Department of Radiology, Neuroradiology

Division, Charlottesville, VA, USA

E. Raz

Department of Radiology, New York University School of Medicine, New York, NY, USA

R. Sanfilippo $\cdot$ R. Montisci

Department of Vascular Surgery, Azienda Ospedaliero Universitaria (A.O.U.), di Cagliari, Italy

E. Raz

Department of Neurology and Psychiatry, Sapienza University of Rome, Rome, Italy elongation, kinking, coiling, dissection, and the side affected by CAD.

Results Fisher's exact test showed a statistically significant association between the ICAD and kinking $(p=0.0089)$ and coiling ( $p=0.0251)$ whereas no statistically significant difference was found with arterial vessel elongation $(p=0.444)$. ICAD was more often seen on the left side compared to the right $(p=0.0001)$. These results were confirmed using both carotid arteries of the same patient as dependent parameter with $p=0.0012,0.0129$, and 0.3323 for kinking, coiling, and elongation, respectively.

Conclusion The presence of kinking and coiling is associated with ICAD.

\section{Keywords Carotid · Dissection}

\section{Introduction}

Internal carotid artery dissection (ICAD) is a rare condition with an annual incidence of 3 cases per 100,000 [1]. ICAD is responsible for $20 \%$ of ischemic stroke in young adults under 45 years old $[2,3]$ and for about $2 \%$ of ischemic strokes overall $[4,5]$. The incidence of ICAD may be underestimated because of the challenges to diagnose this condition [6]. The increasing number of ICAD reports in recent years reflects both a growing familiarity with this complex clinical entity and a significant improvement in the diagnostic tools available to detect this condition [6].

The risk factors for ICAD are still a matter of debate. Several hypotheses have been proposed, including classical vascular risk factors (hypertension, diabetes, smoke, hyperlipidemia, hyper-homocysteinemia), connective tissue abnormalities, major cervical trauma, chiropractic manipulation, and infections [7-14]. The presence of geometrical predisposing factors such as the length of the carotid arteries and/or the 
potential interaction of the carotid arteries with other anatomical structures (elongated temporal styloid process in Eagle syndrome) did not show a significant association with ICAD $[15,16]$. In general, ICAD should be considered as a multifactorial disease with a genetic predisposition, which may cause arterial wall weakness, and environmental factors (hyperextension-rotation trauma) that may trigger the dissection.

Previous publications have shown that the prevalence of vessel tortuosity can reach up to the $18 \%[17,18]$ and that the blood flow in tortuous vessels may be reduced by more than $40 \%$ with an ICA angle of $60^{\circ}$ and by more than $60 \%$ with an ICA angle of $30^{\circ}[19,20]$.

The purpose of this work was to assess whether there is an association between the presence of ICAD and arterial tortuosity in a multi-center cohort of patients.

\section{Materials and methods}

Study design and patient population The Institutional Review Board approved this study. Because of the retrospective nature of this analysis, the IRB granted a waiver of consent. In this work, patients from four cooperating institutions were selected (blinded for peer review) if they had both CT-angiogram (CTA) and MRA confirming the presence of ICAD. The MRI exam was considered positive for ICAD when there was a characteristic crescentic hyper-intense signal in T1-W fat sat images in the arterial wall consistent with an intramural hematoma. CT was considered positive for the presence of ICAD when (a) in the basal scan, the crescent shape sign was detected (b) after contrast material administration the "target sign" was found. Patients were excluded if they presented with bilateral dissections. Demographic data were retrieved and the cause of the dissection (traumatic versuss spontaneous) was recorded.

CTA technique In all four centers, CTA of the cervical carotid arteries was obtained using $60-80 \mathrm{ml}$ of contrast medium (Ultravist 370; Schering, Berlin, Germany) into an antecubital vein, injected at a rate of $4-5 \mathrm{ml} / \mathrm{s}$, followed by $30 \mathrm{ml}$ of saline chase. The precise timing of the CT image acquisition compared to the injection was determined using a bolus-tracking technique: the dynamic monitoring started $6 \mathrm{~s}$ after the beginning of the intravenous injection of contrast material. When the threshold was reached $(+90 \mathrm{HU}$ above the baseline in a region of interest drawn on aortic arch triggered to $\mathrm{CT}$ acquisition), the acquisition was performed in a caudo-cranial direction from the aortic arch to the Circle of Willis. Acquisition parameters were as follows: slice thickness of $1.3 \mathrm{~mm}$, increment $0.6 \mathrm{~mm}$, matrix $512 \times 512$, field of view (FOV) 16 $19 \mathrm{~cm}, 260-300 \mathrm{mAs}$, and $120-140 \mathrm{kV}$.

MRI technique MRI studies were performed using different protocols at each of the four contributing institutions. However, all MRI scans included a T1-weighted sequence with fat suppression (T1-W SPIR-Spectral Presaturation with Inversion Recovery) and an angiographic sequence acquired with Time of flight (TOF) technique. The T1-W SPIR sequence was mandatory for patients to be included in this research study.

Image analysis Two experienced radiologists (XX blinded for peer review, 9 years of experience; YY blinded for peer review, 8 years of experience) blinded to the clinical information and to each other, performed independently the
Fig. 1 Diagram of CTA volume rendered based on the normal course of the ICA (a), elongation (b), kinking (c) and coiling (d)

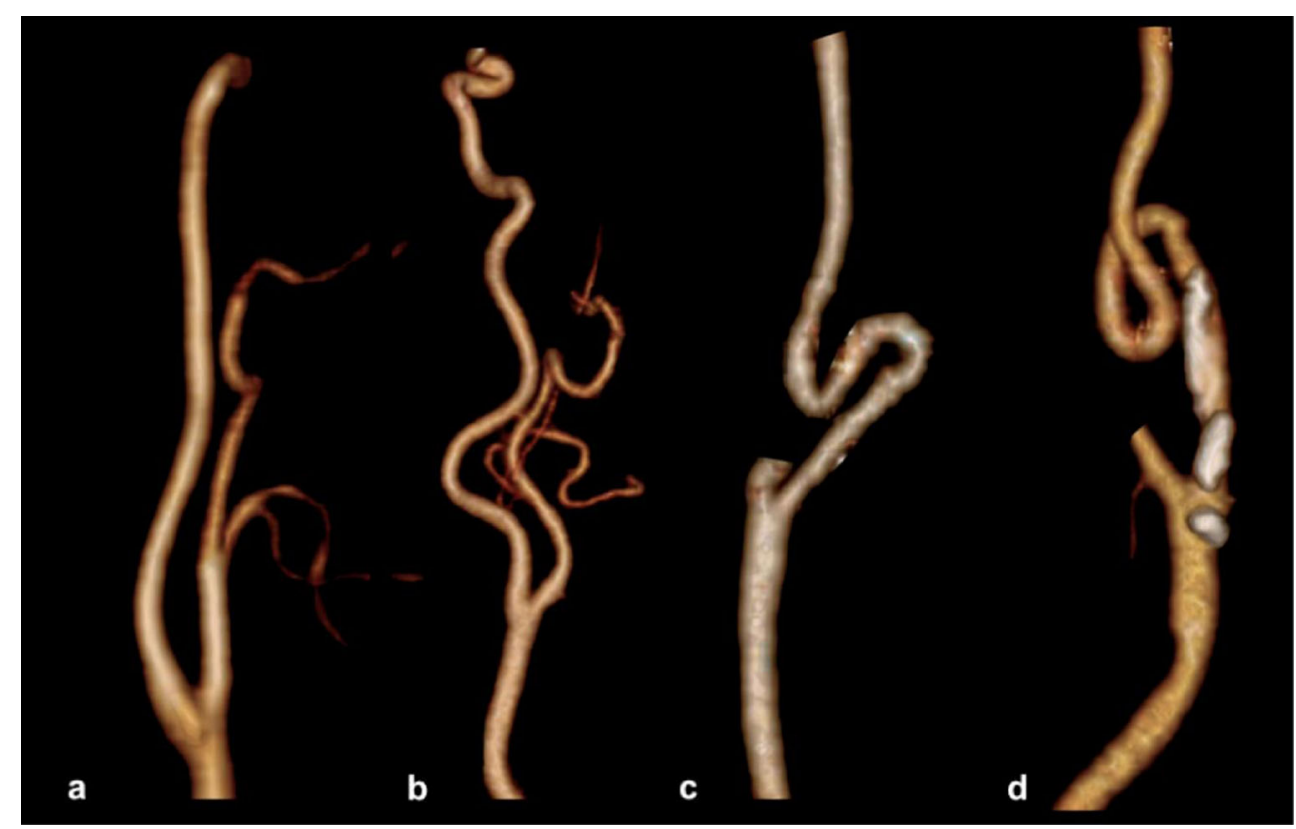


Table 1 Demographic characteristics

\begin{tabular}{llll}
\hline & Traumatic $(n=64)$ & Spontaneous $(n=60)$ & $p$ value \\
\hline Age (years) & $54 \pm 16$ & $59 \pm 15$ & $0.0405^{*}$ \\
Gender (male) & $68.75 \%(44)$ & $48.33 \%(29)$ & $0.0209 *$ \\
Head-neckache & $40.63 \%(26)$ & $30 \%(18)$ & 0.216 \\
Smoker (never) & $43.75 \%(28)$ & $46.67 \%(28)$ & 0.744 \\
Smoker (ex) & $28.13 \%(18)$ & $31.67(19)$ & 0.668 \\
Smoker (current) & $28.13 \%(18)$ & $21.67 \%(13)$ & 0.618 \\
Hypertension & $46.88 \%(30)$ & $43.33 \%(26)$ & 0.692 \\
Diabetes & $20.31 \%(13)$ & $23.33 \%(14)$ & 0.683 \\
Dyslipidemia & $53.13 \%(34)$ & $48.33(29)$ & 0.594 \\
\hline
\end{tabular}

*statisically significant $p$ value

assessment of internal carotid artery morphology. In case of discordant results a third senior reader served as the final adjudicator (ZZ blinded for peer review, 11 years of experience). The tortuosity of the vessels was assessed according to the criteria indicated by Metz et al. [21] and Wiebel and Fields [22].

In these criteria, coiling was diagnosed in the presence of elongation or redundancy of the ICA in a restricted space, causing tortuosity and resulting in an exaggerated S-shaped curve or a circular (or double circular) configuration. Kinking was considered as a variant of coiling (less pronounced or $Z$ shape), where it is visible in an acute angulation of one or more segments of the ICA with an angle between the two segments forming the kink with $60^{\circ}$ or less. Arterial elongation was generically considered as the presence of abnormal vessel length without kinking or coiling with S- or C-shaped tortuosity or ondulation in the course of the ICA (Fig. 1). For the inter-observer analysis, we considered both CT and MR data sets but for the identification of the vessel status (kinking, coiling, elongation) and the need of the third adjudication, the CT dataset were considered.

Statistical analysis The normality of each continuous variable group was tested using Kolmogorov-Smirnov $Z$ test. Continuous data were described as mean $\pm \mathrm{SD}$. Because the normality assumption was verified, unpaired Student's $t$ tests were used to test differences between the groups examined. We created two models of analysis; in the first model, the carotid arteries of both sides were considered as independent units $(n=248)$ for the presence of kinking/coiling/elongation and the Fisher's exact test was applied to test the statistical hypothesis. In the second model, we considered the both carotid arteries of the same patient as dependent parameter in order to have an element controlling for patient in the statistical analysis and we tested the effect of the kinking/coiling/elongation using the McNemar's test. Inter-observer/technique agreement was calculated using the Cohen weighted test. A value $<0$ was considered poor agreement, 0 to 0.2 slight agreement, 0.21 to 0.4 fair agreement, 0.41 to 0.6 moderate agreement, 0.61 to 0.8 substantial agreement, and 0.81 to 1 almost perfect agreement. Statistical analysis was performed with the SPSS 13.0 statistical package (SPSS Inc, Chicago, IL). $P$ values $<0.05$ were regarded as statistically significant, and all $p$ values were calculated using a two-tailed significance level. Graphics were plotted with MedCalc 8.0 software (MedCalc, Mariakerke, Belgium).

\section{Results}

General results This study considered 124 patients ( 72 males, 52 females, age $57 \pm 16$ years, median age 58 years, range 10 89) for a total of 248 carotid arteries analyzed. The most common symptom was head/neckache, reported by $78 \%$ of the subjects (97/123). We found 55/248 arteries showing elongation (prevalence $22.17 \%$ ), 48/248 arteries showing kinking (prevalence 19.35\%), and 22/248 arteries showing coiling (prevalence of $8.9 \%$ ). The total prevalence of arterial tortuosity was therefore $50.4 \%$ with a total number of 125 cases (of 248 carotid arteries). The prevalence of traumatic nature of the dissection was $51.6 \%$ ( $n=64$ cases $)$, and the remaining 60 cases were spontaneous ICAD. The demographic characteristics of the studied patients are shown in Tables 1 and 2 .

ICAD versus tortuosity In the first model we created, by considering the carotid arteries as independent units $(n=$ 248 ), we found a statistically significant association between ICAD and kinking $(p=0.0089)$ and coiling $(p=0.0251)$ whereas no statistically significant difference was found between ICAD and arterial vessel elongation $(p=0.444)$. In the second model, we considered the ICA contralateral to the dissected one in order to have an element controlling for patient, and the McNemar's test showed that there was a

Table 2 Vessel tortuosity versus age, gender, head-neckache

\begin{tabular}{llllll}
\hline Parameter & No anomalies $n=123(\mathrm{~A})$ & Elongation $n=55(\mathrm{~B})$ & Kinking $n=48(\mathrm{C})$ & Coiling $n=22(\mathrm{D})$ & $p$ values \\
\hline Age (years \pm SD) & $53 \pm 16$ & $60 \pm 16$ & $60 \pm 15$ & $61 \pm 16$ & A versus B/C/D<0.05 \\
Gender (male) & $38(52 \%)$ & $14(19 \%)$ & $13(18 \%)$ & $7(11 \%)$ & In all cases $>0.05$ \\
Head-neckache & $42(43 \%)$ & $22(23 \%)$ & $24(25 \%)$ & $9(9 \%)$ & In all cases $>0.05$ \\
\hline
\end{tabular}


Table 3 Inter-observer agreement with $\mathrm{CT}$ and MR-kappa value analysis

Standard error is given between parentheses

$N C$ not calculable

\begin{tabular}{lllll}
\hline & CT observer 1 & CT observer 2 & MR observer 1 & MR observer 2 \\
\hline CT observer 1 & NC & $0.873(0.047)$ & $0.828(0.046)$ & $0.834(0.047)$ \\
CT observer 2 & & NC & $0.764(0.046)$ & $0.761(0.046)$ \\
MR observer 1 & & & NC & $0.789(0.047)$ \\
MR observer 2 & & & NC \\
\hline
\end{tabular}

statistically significant difference for the presence of kinking between dissected and non-dissected side $(p=0.0012)$ and also for the coiling ( $p=0.0129)$ whereas no statistically significant difference was found for the elongation $(p=0.3323)$. We also found that in the 124 left carotid arteries, there were 78 ICADs whereas in the 124 right carotids, there were only 47 ICADs. This difference was statistically significant $(p=$ 0.0001 , OR 2.8).

Cohen kappa analysis Results from the Cohen kappa analysis are summarized in the Table 3. We found that the best concordance for the morphology of the arteries was observed with CT (kappa value 0.873 ). However, in the other comparisons, the kappa values were good to excellent (CT versus MR, MR versus MR). In the Table 4, the summary for the $\mathrm{CT}$ (observer 1 and observer 2 ) is given. The 2 readers agreed in 219 cases (88.3\%); the adjudicator was necessary in 29 cases (11.7\%).

\section{Discussion}

ICAD is an uncommon condition, and the underlying cause that determines the dissection of the intima layer with the development of the intra-mural hematoma remains still debated. It is possible to classify two main types of ICAD: traumatic and spontaneous [23]. Several classic cardiovascular risk factors are associated with ICAD (smoking, diabetes, dyslipidemia) as well as other pathologies that affect the carotid artery wall (Marfan's syndrome, Ehlers-Danlos syndrome, fibromuscular dysplasia) because of the damage that determine to the vessel's wall $[7-11,23,24]$.
Carotid tortuosity is not uncommon in normal subjects, but its role in cerebrovascular diseases is not well defined. In our study population, we found a prevalence of $22.17 \%$ for arterial elongation, $19.35 \%$ for kinking, and $8.9 \%$ for coiling with a total prevalence of arterial tortuosity of $50.4 \%$. This prevalence is superior to those reported in previously published papers. In the work by Pelkonen et al. [16], published in the 2003, the authors found a prevalence of arterial tortuosity of $23 \%$. The difference between this study and our study can be explained by two reasons. First, Pelkonen et al. [16] considered only kinking and coiling whereas we also included the elongation of the internal carotid arteries. By considering only the kinking and coiling, the prevalence in our population is $28.25 \%$, which is close to the $23 \%$ reported by Pelkonen et al. [16] and quite similar to the $29 \%$ found by Ozdoba et al. Second, the average age of our study population was 56 years whereas it was 46.8 years in the work by Pelkonen et al. [16]. The "age-effect" in our cohort can explain an increased prevalence of arterial tortuosity because there is an increased prevalence of arterial tortuosity in elderly subjects [25].

We found a statistically significant association between ICAD and kinking ( $p=0.0089)$ and coiling $(p=0.0251)$ but no statistically significant association with arterial elongation $(p=0.444)$. These results are consistent with the results reported by Babour et al. [26] who analyzed 13 cases of patients with ICAD and 108 without ICAD and found a significant correlation between arterial tortuosity and ICAD. Our study differs from the Babour et al.'s [26] article because of the number of ICAD patients in our cohort $(n=124)$ and the technology used (CTA/MRA for us versus DSA for Babour et al. [26]).
Table 4 Inter-observer agreement for CT summary ${ }^{\mathrm{a}}$

${ }^{\text {a }}$ In this table $4 \times 4$ the interobserver agreement analysis is given. The perfect correspondence is observed in 219 cases whereas in the other 29 the adjudicator (third senior observer) was necessary

\begin{tabular}{llllll}
\hline & \multicolumn{2}{l}{ CT observer 1 } & & \\
\cline { 2 - 5 } CT observer 2 & 1 & 2 & 3 & 4 & \\
\hline 1 & 89 & 2 & 0 & 0 & $91(36.7 \%)$ \\
2 & 7 & 49 & 9 & 3 & $68(27.4 \%)$ \\
3 & 1 & 2 & 66 & 3 & $72(29.0 \%)$ \\
4 & 0 & 1 & 1 & 15 & $17(6.9 \%)$ \\
& $97(39.1 \%)$ & $54(21.8 \%)$ & $76(30.6 \%)$ & $21(8.5 \%)$ & 248 \\
\hline
\end{tabular}


A potential explanation of the association between arterial tortuosity and ICAD may rely in the change in blood flow hemodynamics that can locally disrupt the endothelial architecture leading to a widening of tight junctions and vessel wall inflammation with the release of inflammatory agents determining alteration in the vasomotion and predisposing to ICAD [27]. In particular, the presence of severe kinking may play a role as demonstrated by Baracchini et al. [27]. The association between ICAD and coiling remains unexplained.

Another finding we detected was a significantly more frequent occurrence of ICAD on the left side $(p=0.0001$, OR 2.8). This unexpected difference may be contributed to by anatomical differences, namely the fact that the left internal carotid artery originates directly from the aortic arch while the right carotid artery originates from the innominate artery.

We acknowledge several limitations to our study. First, this was a multi-centric study and different types of CT/MR scanners were used. However, we think that this is a minor limitation because in all cases the same MR sequences were used and the CT protocols were quite similar. Second, this is a retrospective study but we do not think that the association between ICAD and arterial tortuosity should be affected by the retrospective design of our study.

\section{Conclusion}

In conclusion, the results of our study suggest that the presence of kinking and coiling represent morphological association with ICAD. ICAD occurs more frequently on the left side.

Ethical standards and patient consent We declare that all human and animal studies have been approved by the Institutional Review Board of the Azienda Ospedaliero Universitaria Department of Radiology and have therefore been performed in accordance with the ethical standards laid down in the 1964 Declaration of Helsinki and its later amendments. Patient consent was waived due to the retrospective nature of this study.

Conflict of interest We declare that we have no conflict of interest.

\section{References}

1. Giroud M, Dumas R, Becker F (1993) Spontaneous vertebral artery dissection initially revealed by a pain in one upper arm. Stroke 33: 480-481

2. Bevan H, Sharma K, Bradley W (1990) Stroke in young adults. Stroke 21:382-386

3. Dziewas R, Konrad C, Dräger B (2003) Cervical artery dissection clinical features, risk factors, therapy and outcome in 126 patients. J Neurol 250:1179-1184

4. Schievink WI, Mokri B, Garrity JA, Nichols DA, Piepgras DG (1993) Ocular motor nerve palsies in spontaneous dissections of the cervical internal carotid artery. Neurology 43:1938-1941
5. Chancellor AM, Glasgow GL, Ockelford PA, Johns A, Smith J (1989) Etiology, prognosis, and hemostatic function after cerebral infarction in young adults. Stroke 20:477-482

6. Micheli S, Paciaroni M, Corea F, Agnelli G, Zampolini M, Caso V (2010) Cervical artery dissection: emerging risk factors. Open Neurol J 4:50-5

7. Pezzini A, Caso V, Zanferrari C (2006) Arterial hypertension as risk factor for spontaneous cervical artery dissection. A case-control study. J Neurol Neurosurg Psychiatry 77:95-7

8. Caso V, Paciaroni M, Corea F (2004) Recanalization of cervical artery dissection: influencing factors and role in neurological outcome. Cerebrovasc Dis 17:93-7

9. Baumgartner RW, Arnold M, Baumgartner I (2001) Carotid dissection with and without ischemic events: local symptoms and cerebral artery findings. Neurology 57:827-32

10. Gallai V, Caso V, Paciaroni M (2001) Mild hyperhomocyst(e)inemia: a possible risk factor for cervical artery dissection. Stroke 32:714-8

11. Pezzini A, Del Zotto E, Archetti S (2005) Plasma homocysteine concentration, C677TMTHFR genotype, and 844ins68bp CBS cerebral artery dissection, 1st edn. Karger, Switzerland, pp 16-29

12. Ulbricht D, Diederich J, Hermann-Le T, Metz RJ, Macian F, Pierard GE (2004) Cervical artery dissection. An atypical presentation with Ehrles-Danlos like collagen pathology? Neurology 63:1708-1710

13. Arauz A, Hoyos L, Cantu C (2007) Mild hyperhomocysteinemia and low folate concentrations as risk factors for cervical arterial dissection. Cerebrovasc Dis 23:210-4

14. Tzourio C, Cohen A, Lamisse N, Biousse V, Bousser MG (1997) Aortic root dilatation in patients with spontaneous cervical artery dissection. Circulation 95:2351-2353

15. Heckmann JG, Tomandl B, Duhm C, Stefan H, Neundörfer B (2000) Collet-Sicard syndrome due to coiling and dissection of the internal carotid artery. Cerebrovasc Dis 10(6):487-8

16. Pelkonen O, Tikkakoski T, Leinonen S, Pyhtinen J, Lepojärvi M, Sotaniemi K (2003) Extracranial internal carotid and vertebral artery dissections: angiographic spectrum, course and prognosis. Neuroradiology 45:71-7

17. Saba L, Mallarini G (2010) Correlation between kinking and coiling of the carotid arteries as assessed using MDCTA with symptoms and degree of stenosis. Clin Radiol 65:729-34

18. Macchi C, Gulisano M, Giannelli F, Catini C, Pratesi C, Pacini P (1997) Kinking of the human internal carotid artery: a statistical study in 100 healthy subjects by echocolor Doppler. J Cardiovasc Surg 38:629-37

19. Derrick JR, Estess M, Williams D (1965) Circulatory dynamics in kinking of the carotid artery. Surgery $58: 381-3$

20. Smith RB III, Dodson TF (1990) Treatment of carotid kinks, carotid coils, and fibromuscular dysplasia. In: Veith FJ (ed) Current critical problems in vascular surgery. Quality Medical Publishing, Inc, St. Louis, pp 398-406

21. Metz H, Murray-Leslie RM, Bannister RG, Bull JWD, Marshall J (1961) Kinking of the internal carotid artery. Lancet 191:424-6

22. Weibel J, Fields WS (1965) Tortuosity, coiling and kinking of the internal carotid artery. II. Relationship of morphological variation to cerebrovascular insufficiency. Neurology 15:462-8

23. Schievink WI (2001) Spontaneous dissection of the carotid and vertebral arteries. N Engl J Med 344:898-906

24. Trotter SE, Olsen EG (1991) Marfan's disease and Erdheim's cystic medionecrosis: a study of their pathology. Eur Heart J 12:83-7

25. Ciccone MM, Sharma RK, Scicchitano P, Cortese F, Salerno C, Berchialla P, Frasso G (2014) Dolichocarotids: echo-color Doppler evaluation and clinical role. J Atheroscler Thromb 21:56-63

26. Barbour PJ, Castaldo JE, Rae-Grant AD, Gee W, Reed JF 3rd, Jenny D, Longennecker J (1994) Internal carotid artery redundancy is significantly associated with dissection. Stroke 25:1201-6

27. Baracchini C, Farina F, Tonello S, Citton V, Meneghetti G, Ballotta E, Manara R (2013) Endothelial dysfunction in carotid elongation. J Neuroimaging 23:18-20 